\title{
Decreased abundance of Akkermansia muciniphila after adrenocorticotropic hormone therapy in patients with West syndrome
}

Lu Xu

Zhejiang University School of Medicine https://orcid.org/0000-0003-2470-8460

Feng Gao ( $\triangle$ peakgf@126.com )

Shanshan Mao

Zhejiang University School of Medicine

Lihua Jiang

Zhejiang University School of Medicine

Chao Song

Zhejiang University School of Medicine

Congying Zhao

Zhejiang University School of Medicine

Dandan Chen

Zhejiang University School of Medicine

\section{Research article}

Keywords: West syndrome, gut microbiota, ACTH therapy, Akkermansia muciniphila

Posted Date: July 10th, 2019

DOI: https://doi.org/10.21203/rs.2.11198/v1

License: (c) (1) This work is licensed under a Creative Commons Attribution 4.0 International License.

Read Full License

Version of Record: A version of this preprint was published at BMC Microbiology on April 23rd, 2021. See the published version at https://doi.org/10.1186/s12866-021-02189-z. 


\section{Abstract}

Background West syndrome (WS) is a severe epileptic encephalopathy of infancy. The high degree of effectiveness of adrenocorticotropic hormone (ACTH) therapy indicates that the hypothalamic-pituitaryadrenal (HPA) axis may be involved in WS, and there is substantial evidence that the gut microbiota (GM) communicates with the brain via the HPA axis. The aims of this study were to determine and compare the diversity of the GM in infants with WS and healthy infants, and in patients with WS before and after ACTH therapy. Results A total of 19 infants with WS and 24 healthy infants in the same age range (3-13 months) were enrolled. Fecal samples were collected and DNA was extracted and sequenced on the Illumina HiSeq platform. The between-group differences in Chao1 index (a diversity) and the abundances of GM genera were analyzed using the Wilcoxon signed-rank test; graphs were plotted using R software. There were no significant differences in the top four dominant GM genera between patients with WS and healthy infants; however, the abundance of the species Akkermansia muciniphila was significantly $(\mathrm{P}=$ 0.017) higher in the WS group than in healthy infants. After 2 weeks of ACTH therapy, the abundance of this bacteria was significantly $(P=0.022)$ and dramatically decreased. Conclusion The abundance of $A$. muciniphila was significantly higher in patients with WS than healthy infants. Further research is needed to clarify the relationship between A. muciniphila and WS pathogenesis.

\section{Background}

West syndrome (WS) is a severe epileptic encephalopathy of infancy, characterized by infantile spasms, hypsarrhythmia (as recorded on interictal electroencephalographs [EEGs]), and neurodevelopmental delay. Owing to poor responses to conventional antiepileptic drugs, adrenocorticotropic hormone (ACTH), vigabatrin, and oral corticosteroids are recommended by guidelines and reviews ${ }^{[1-3]}$ as the first-line drug treatment for WS. ACTH therapy is a highly effective treatment for infantile spasms. A possible mechanism underlying WS involves increased levels of corticotropin-releasing hormone ${ }^{[4]}$ leading to elevated neuronal excitability and infantile spasms, indicating that the hypothalamic-pituitary-adrenal (HPA) axis may be involved in WS.

The gut microbiota (GM) communicates with the brain via the vagus nerve, HPA axis, gut immune system, and neurotransmitters synthesized by intestinal bacteria. Research over the last decade on the brain-gut-microbiota axis, which has been shown to involve various regions of the nervous system, has mainly focused on psychiatric and neurologic pathologies, including chronic pain ${ }^{[5]}$, autism spectrum disorder ${ }^{[6]}$, Parkinson's disease ${ }^{[7]}$, and multiple sclerosis ${ }^{[8]}$. Additionally, in recent years, the GM has been found to have an important role in the treatment of refractory epilepsy, especially regarding its effect in mediating the antiseizure effects of the ketogenic diet ${ }^{[9,10]}$.

WS may be influenced by communication between the GM and the brain via the HPA axis. To gain increased understanding of the mechanisms underlying the therapeutic effectiveness of ACTH for WS from a new perspective, this study investigated the abundance of GM genera in patients with WS and 
evaluated differences in the GM between patients with WS and healthy infants (HIs), and between patients before and after short-term ACTH treatment.

\section{Methods}

\section{Patients, fecal sample collection, and ACTH therapy}

A total of 19 infants with WS (aged 3-13 months) were enrolled at the Zhejiang University School of Medicine from August to December 2018. WS diagnosis was confirmed based on three typical characteristics: spasms, neurodevelopmental delay, and hypsarrhythmia on EEG. The control group comprised $24 \mathrm{His}$ in the same age range (3-13 months). No antibiotics or probiotics were used for at least 3 months before the study. Patient data were collected prospectively, including: age, sex, etiology, antiepileptic drug use, and treatment effects.

Fecal samples were collected twice (before and after ACTH therapy). If fecal samples could not be processed immediately, they were transferred to a $-80^{\circ} \mathrm{C}$ freezer within $24 \mathrm{~h}$.

Based on the instructions for ACTH therapy, low-dose (20-25 U/day) natural ACTH, which was extracted from porcine pituitary glands, was administered by intravenous injection for $>8 \mathrm{~h} /$ day for 2 weeks. During the treatment period, before the second feces collection, use of other medicines (such as antiepileptic drugs, antibiotics, or probiotics) remained fixed (with the same regimen of antiepileptic drugs throughout and no antibiotics or probiotics started before the second feces collection had been completed).

DNA extraction, library construction, and sequencing

Genomic DNA was extracted from the fecal samples using a DNA extraction kit (DP328, Tiangen Biotech Co., Ltd., Beijing, China), according to the manufacturer's instructions. The hypervariable V3-V4 region of the $16 \mathrm{~S}$ rRNA gene was amplified using region of interest-specific primers with overhang adapters (341F: 5- $\mathbb{C} T A C G G G N G G C W G C A G-3$ and 805R: 5- GACT ACHVGGGTATCT $\forall T \mathbb{C}-3$ ). Indices and sequencing adapters were then attached using the Nextera XT Index Kit. An Agilent SureCycler 8800 Thermal Cycler was used for PCR, according to the manufacturer's instructions. The protocol involved 25 cycles of denaturation at $95^{\circ} \mathrm{C}$ for $30 \mathrm{~s}$, annealing at $55^{\circ} \mathrm{C}$ for $30 \mathrm{~s}$, and extension at $72^{\circ} \mathrm{C}$ for $30 \mathrm{~s}$, followed by a final elongation at $72^{\circ} \mathrm{C}$ for $5 \mathrm{~min}$. Samples were purified by magnetic bead DNA isolation and electrophoresis was conducted as part of the quality control protocol. Libraries were sequenced and PE300 raw data in FASTQ format were obtained using the Illumina MiSeq platform (Illumina, Inc., San Diego, CA, USA).

To ensure the accuracy of the results, sequences were further processed to obtain high-quality sequences for analysis as follows. First, primer sequences were removed using Cutadapt (version 1.11) and the resultant paired-end reads were assembled using PANDAseq (version 2.9). Reads with an average quality score $<20$, ambiguous bases, length $<300$ or $>480 \mathrm{bp}$, or chimeric sequences were excluded. The 
remaining sequences were assigned to OTUs, using QIIME, based on a similarity of $97 \%$, and classified using the Ribosomal Database Project (RDP) Classifier ${ }^{[17]}$.

\section{Data analysis}

Differences in the Chao1 index (a diversity) and the abundances of GM genera were compared between patients with WS and HIs, and between WS patients before (B1) and after (A1) ACTH therapy, using the Wilcoxon signed-rank test. Graphs were plotted using R software (version 3.4.3). Subsequently, the functional profile predictions of the $B 1, A 1$, and $\mathrm{HI}$ groups were analyzed using PICRUSt. A principal coordinate analysis (PCoA) was also conducted to identify the degree of similarity between genera.

\section{Results}

\section{Clinical improvement after ACTH therapy}

First, we evaluated 19 patients with WS and $24 \mathrm{HI}$ controls in the same age range (3-13 months). Patients with WS were assessed before (B1) and after (A1) ACTH therapy. Following ACTH therapy, 10 $(52.6 \%)$ patients were seizure free, $8(42.1 \%)$ had a $60 \%-90 \%$ reduction in seizure frequency, and only one patient was unresponsive. However, regarding EEG-based outcomes, 4 (21.1\%) patients had persistent hypsarrhythmia at the end of treatment; one patient had no follow-up EEG.

Owing to respiratory infection caused by ACTH therapy, three patients required antibiotics (two were seizure free and the third had a $60 \%-90 \%$ reduction in seizure frequency) and fecal samples were obtained before antibiotic treatment (before the end of the planned 2-week treatment period). For the remaining patients with WS, fecal sampling was complete on day 14 or 15 . There were $10(52.6 \%)$ patients with WS who did not take antiepileptic drugs before the study.

3.2. Differences in GM between patients with WS and HIs, and between patients before (B1) and after (A1) ACTH therapy

After filtering and quality control, the mean high-quality bacterial DNA read length was $34853 \mathrm{bp}$ (range: 29129-37847 bp). Reads were assigned to operational taxonomic units (OTUs) based on similarity scores of $97 \%$.

Analysis of the Chao1 index revealed higher GM diversity in the B1 group, compared with the HI group, although the difference was not significant $(P=0.0991)$ (Figure 1). The top four genera in the B1 group were Bifidobacterium, Bacteroides, Veillonella, and Escherichia/Shigella (Figure 2). Patients with WS and Hls were in the same age range (3-13 months) and had experienced different feeding patterns. There was no significant difference in the top four genera of GM between the B1 and HI groups. However, the abundance of Akkermansia muciniphila was significantly higher in the B1 group compared with the $\mathrm{HI}$ group $(P<0.05)$ (Figure 2), and the Akkermansia abundance was significantly $(P=0.022)$ and dramatically decreased after ACTH therapy. Among the nine patients with WS who exhibited a change in 
Akkermansia abundance after ACTH therapy, eight (88.9\%) exhibited varying degrees of decrease in Akkermansia abundance (Figure 3). Moreover, the seizure frequency reduced in all the patients who exhibited a decrease in Akkermansia abundance.

There was no difference in diet or medication use between the A1 and B1 groups, except for whether ACTH therapy was administered, and PCoA indicated that the GM genera detected did not differ between these groups (Figure 4).

Functional analysis of the microbiota was conducted using PICRUSt. Comparisons of data from the B1 and $\mathrm{HI}$ groups revealed significance differences in amino acid-related enzymes $(P=0.028)$ and pantothenate and CoA biosynthesis $(P=0.024)$.

\section{Discussion}

There is clear evidence of a bidirectional relationship between the GM and the brain, termed the brain-gutmicrobiota axis, which is associated with numerous health disorders ${ }^{[5-8]}$. In recent years, the GM has been found to impact neuronal activity, leading to the development of epilepsy ${ }^{[9]}$. We selected a specific epilepsy syndrome (WS) to compare the GM diversity between patients and HIs, and to compare the GM before and after treatment.

In our study, there were no differences in the abundances of the top four most abundant GM genera between the patients with WS and HIs, indicating no obvious GM dysbiosis in patients with WS. However, the abundance of the genus Akkermansia was significantly higher in the patients with WS compared with the HI group. Influences on the GM include geography, diet, stress, physiological stress caused by exercise, critical illness, and medications, such as agents that cause gastric acid suppression and antibiotics $^{[11]}$. By keeping factors other than ACTH therapy as constant as possible, we found that the abundance of Akkermansia was significantly and dramatically decreased following treatment with ACTH.

A. muciniphila (phylum, Verrucomicrobia) in the human GM is involved in conditions including glucose metabolism, obesity, and inflammatory bowel disease ${ }^{[12,13]}$. Several beneficial functions of A. muciniphila for host health have been reported, including increased gut barrier integrity, increased mucus thickness, and beneficial metabolic and immune responses ${ }^{[14]}$; however, the relationship between Akkermansia and epilepsy remains unclear, and previous investigations have generated conflicting results. Olson et al ${ }^{[9]}$ found that Akkermansia mediates ketogenic diet-induced protection against 6-Hz-induced seizures in a mouse model of refractory epilepsy. In contrast, we found that Akkermansia abundance was higher in patients with WS compared with HIs. An important explanation for this discrepancy may be that these different effects of Akkermansia on WS patients are mediated by different immune-related mechanisms. This hypothesis is based on the specific causes of the onset of WS and poor response to antiepileptic treatment, which are indicative of an opposite effect of Akkermansia underlying WS. Cekanaviciute et al. 
reported that Akkermansia can drive T lymphocyte differentiation toward the proinflammatory interferon $\mathrm{y}+$ Th1 phenotype in vitro, and that increased Akkermansia abundance in vivo may directly or indirectly exacerbate the inflammatory effects of multiple sclerosis ${ }^{[15]}$.

Pediatricians use ACTH therapy as a first-line treatment for WS and it was effective in our patients; however, the mechanisms underlying the effectiveness of ACTH for WS therapy remain unclear. The blood-brain barrier is thought to be impermeable to ACTH, hence the therapeutic effects may not be mediated directly ${ }^{[16]}$. We hypothesize that exogenous ACTH directly influences the dynamics of the HPA axis, leading to alterations in the GM. Although the underlying reasons why some patients' seizures can be controlled by ACTH remain unknown, our results provide an important addition to the literature regarding the pathogenesis of WS and the mechanism underlying the therapeutic effectiveness of ACTH.

Our study had several limitations. First, the sample size was small. A larger sample size would increase the statistical power, which would increase the validity of the conclusions based on the statistical analysis of the data regarding the relationships between the abundances of GM genera and WS. Second, the study was short term, and long-term follow-up is required to comprehensively evaluate the GM alterations in patients with WS. Finally, the mechanism underlying the effect of ACTH on GM when seizures were controlled is unknown. Given these limitations, further studies are recommended, and we intend to conduct research with a larger sample size and simultaneously conduct an association analysis to investigate Akkermansia-related immunological indicators.

\section{Conclusions}

Overall, our data indicate that the abundance of Akkermansia differed significantly between patients with WS and HIs, and between patients before and after ACTH therapy. Further research to fully elucidate the relationships between the GM and WS is currently being designed. These findings have potential to provide the foundation for a new direction of exploration of the pathogenesis of WS.

\section{Abbreviations}

ACTH Adrenocorticotropic hormone

WS West syndrome

GM Gut microbiota

EEG Electroencephalograph

HPA Hypothalamic-pituitary-adrenal axis

OTUs Operational taxonomic units

PCoA Principal coordinate analysis 
PCR Polymerase Chain Reaction

RDP Ribosomal Database Project

\section{Declarations}

\section{Acknowledgements}

We thank the members of JingBai Biological Laboratory who helped with the study.

\section{Authors' contributions}

All authors have read and approved the manuscript. LX and FG designed the study; LX and CZ performed the study; LJ, SM, and CS contributed to collecting, processing, and analyzing samples; LJ, SM, and CZ contributed to the diagnoses; LX and CS analyzed the data; LX and FG wrote the manuscript.

\section{Ethics approval and consent to participate}

The study was approved by the ethics committee at Children's Hospital of Zhejiang University School of Medicine. We have obtained written informed consent from a parent or guardian for participants.

\section{Competing interests}

The authors declare that they have no competing interests.

\section{Funding}

This work was supported by National Natural Science Foundation of China (Grant No.81741076 81801490冈81501084), Zhejiang Provincial Natural Science Foundation of China.(Grant No.LQ17H090004).

Consent for publication

Not applicable.

\section{References}

[1] D'alonzo R, Rigante D, Mencaroni E, et al. West Syndrome: A Review and Guide for Paediatricians[J]. Clin Drug Investig, 2018, 38(2): 113-124.

[2] Wilmshurst J M, Gaillard W D, Vinayan K P, et al. Summary of recommendations for the management of infantile seizures: Task Force Report for the ILAE Commission of Pediatrics[J]. Epilepsia, 2015, 56(8): 1185-97. 
[3] Go C Y, Mackay M T, Weiss S K, et al. Evidence-based guideline update: medical treatment of infantile spasms. Report of the Guideline Development Subcommittee of the American Academy of Neurology and the Practice Committee of the Child Neurology Society[J]. Neurology, 2012, 78(24): 1974-80.

[4] Baram T Z. Models for infantile spasms: an arduous journey to the Holy Grail[J]. Ann Neurol, 2007, 61(2): 89-91.

[5] Amaral F A, Sachs D, Costa V V, et al. Commensal microbiota is fundamental for the development of inflammatory pain[J]. Proc Natl Acad Sci U S A, 2008, 105(6): 2193-7.

[6] Vuong H E, Hsiao E Y. Emerging Roles for the Gut Microbiome in Autism Spectrum Disorder[J]. Biol Psychiatry, 2017, 81(5): 411-423.

[7] Sampson T R, Debelius J W, Thron T, et al. Gut Microbiota Regulate Motor Deficits and Neuroinflammation in a Model of Parkinson's Disease[J]. Cell, 2016, 167(6): 1469-1480 e12.

[8] Mowry E M, Glenn J D. The Dynamics of the Gut Microbiome in Multiple Sclerosis in Relation to Disease[J]. Neurol Clin, 2018, 36(1): 185-196.

[9] Olson C A, Vuong H E, Yano J M, et al. The Gut Microbiota Mediates the Anti-Seizure Effects of the Ketogenic Diet[J]. Cell, 2018, 174(2): 497.

[10] Xie G, Zhou Q, Qiu C Z, et al. Ketogenic diet poses a significant effect on imbalanced gut microbiota in infants with refractory epilepsy[J]. World J Gastroenterol, 2017, 23(33): 6164-6171.

[11] Cresci G A, Bawden E. Gut Microbiome: What We Do and Don't Know[J]. Nutr Clin Pract, 2015, 30(6): 734-46.

[12] Cani P D, De Vos W M. Next-Generation Beneficial Microbes: The Case of Akkermansia muciniphila[J]. Front Microbiol, 2017, 8: 1765.

[13] Rajilic-Stojanovic M, Shanahan F, Guarner F, et al. Phylogenetic analysis of dysbiosis in ulcerative colitis during remission[J]. Inflamm Bowel Dis, 2013, 19(3): 481-8.

[14] Ottman N, Geerlings S Y, Aalvink S, et al. Action and function of Akkermansia muciniphila in microbiome ecology, health and disease[J]. Best Pract Res Clin Gastroenterol, 2017, 31(6): 637-642.

[15] Cekanaviciute E, Probstel A K, Thomann A, et al. Multiple Sclerosis-Associated Changes in the Composition and Immune Functions of Spore-Forming Bacteria[J]. mSystems, 2018, 3(6).

[16] Swann J W, Moshe S L: On the Basic Mechanisms of Infantile Spasms, Th, Noebels J L, Avoli M, Rogawski M A, Olsen R W, Delgado-Escueta A V, editor, Jasper's Basic Mechanisms of the Epilepsies, Bethesda (MD), 2012. 
[17] Wang Q, Garrity G M, Tiedje J M, et al. Naive Bayesian classifier for rapid assignment of rRNA sequences into the new bacterial taxonomy[J]. Appl Environ Microbiol, 2007, 73(16): 5261-7.

\section{Tables}

Due to technical limitations, Table 1 has been placed in the Supplementary Files section.

\section{Figures}

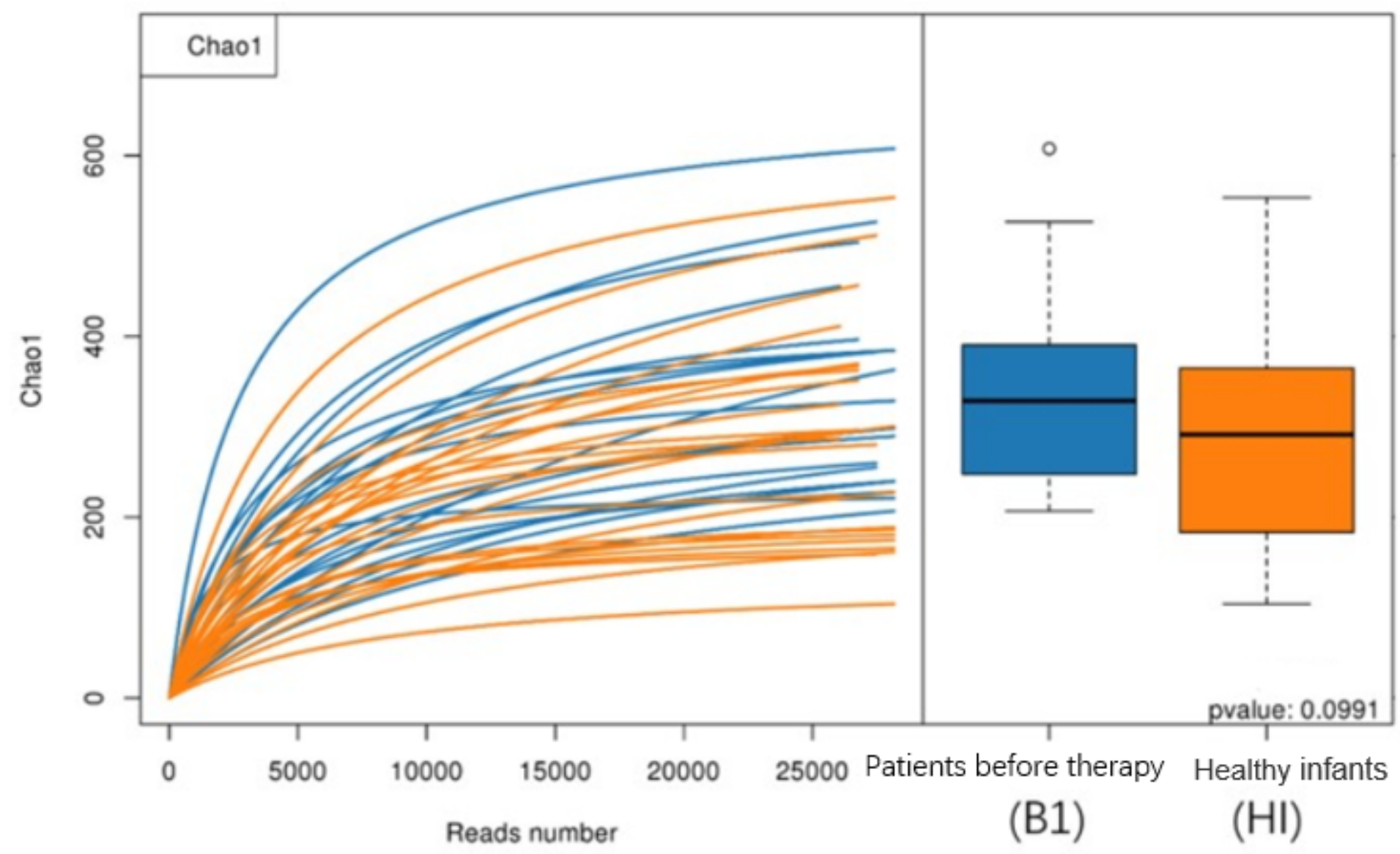

\section{Figure 1}

Differences in GM Chao1 index (a diversity) between patients with WS before therapy (B1; blue) and healthy infants (HIs; orange) evaluated using the Wilcoxon signed-rank test. GM diversity was higher in the B1 group than the HI group, although the difference was not significant $(P=0.0991)$. 


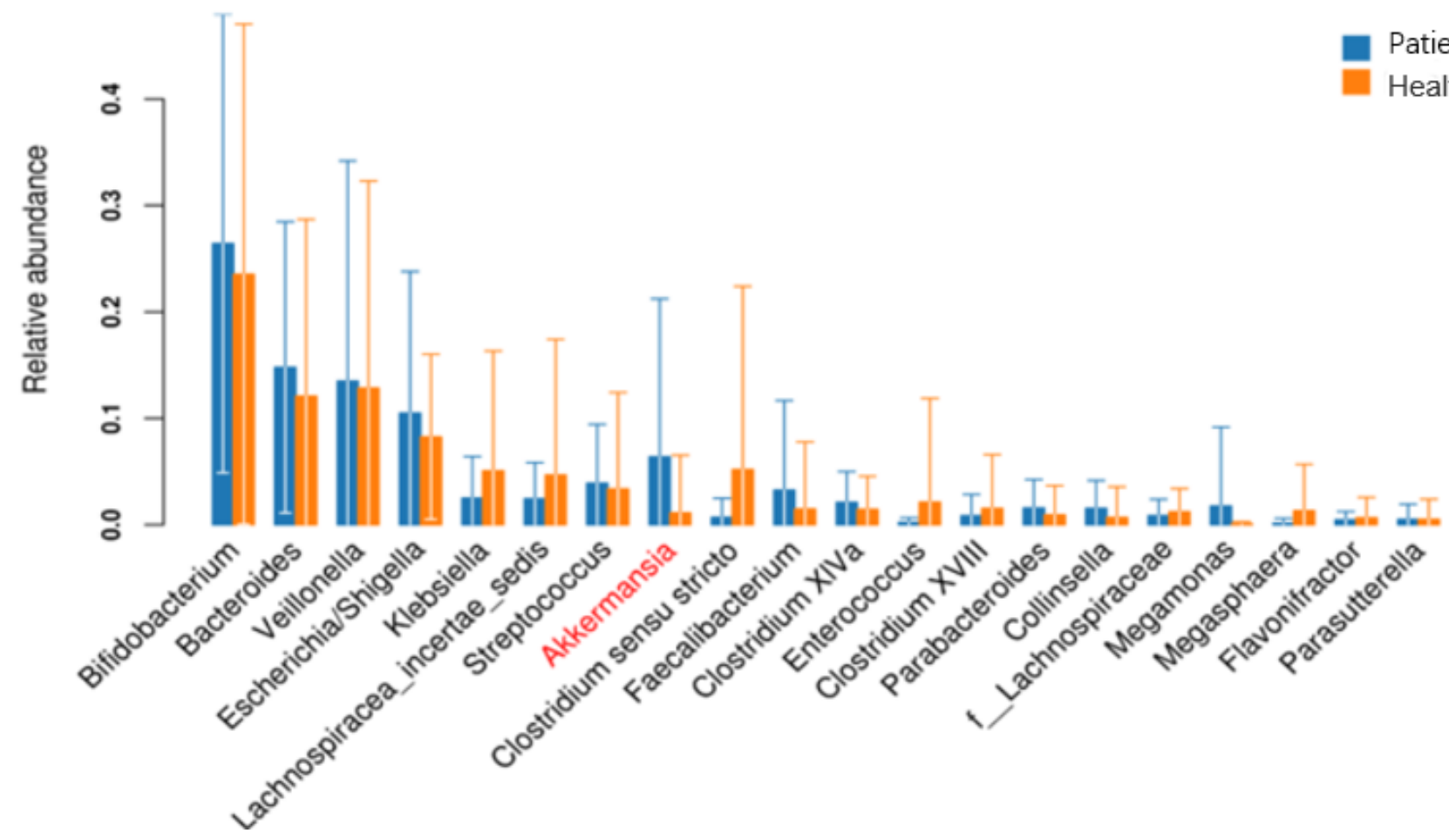

Figure 2

Differences in the top 20 genera between patients with WS before treatment (B1) and healthy infants (HIs) evaluated using the Wilcoxon signed-rank test. A genus that exhibited a significant $(P=0.017)$ difference (Akkermansia) is indicated in red. Akkermansia abundance was significantly higher in the B1 group than the HI group. Error bars show \pm standard error.

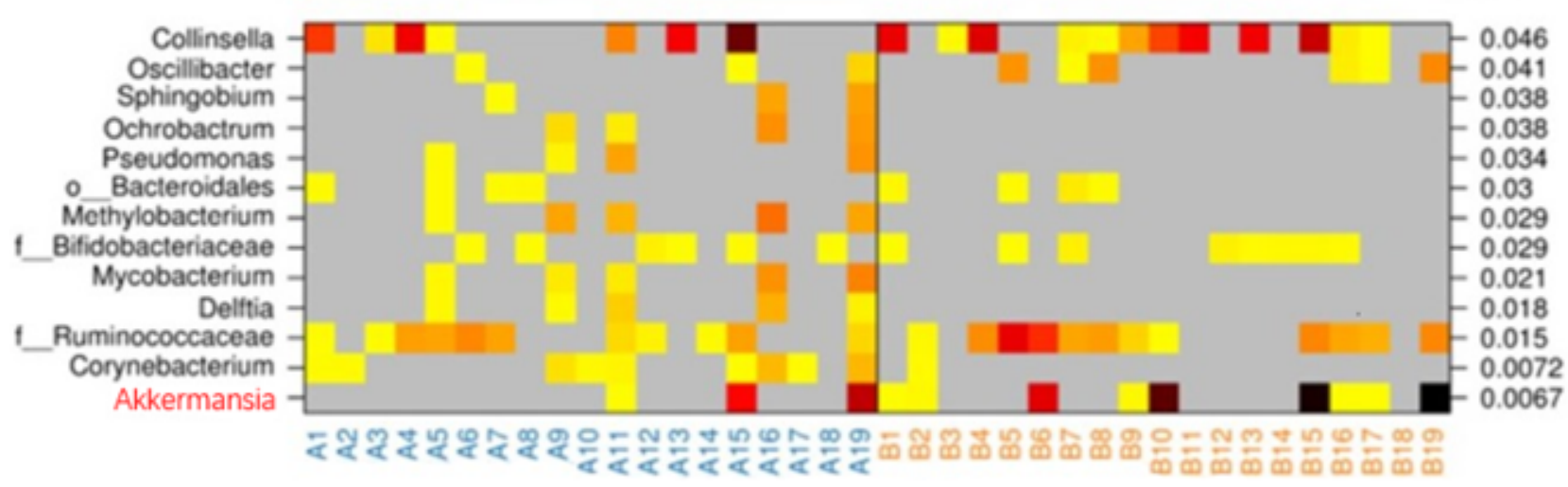

Figure 3

Differences in genera between individual patients with WS before (B1) and after (A1) ACTH therapy. The higher the abundance of the genus, the darker the color. Overall, the abundance of Akkermansia was significantly $(P=0.022)$ decreased after ACTH therapy (Wilcoxon signed-rank test). 


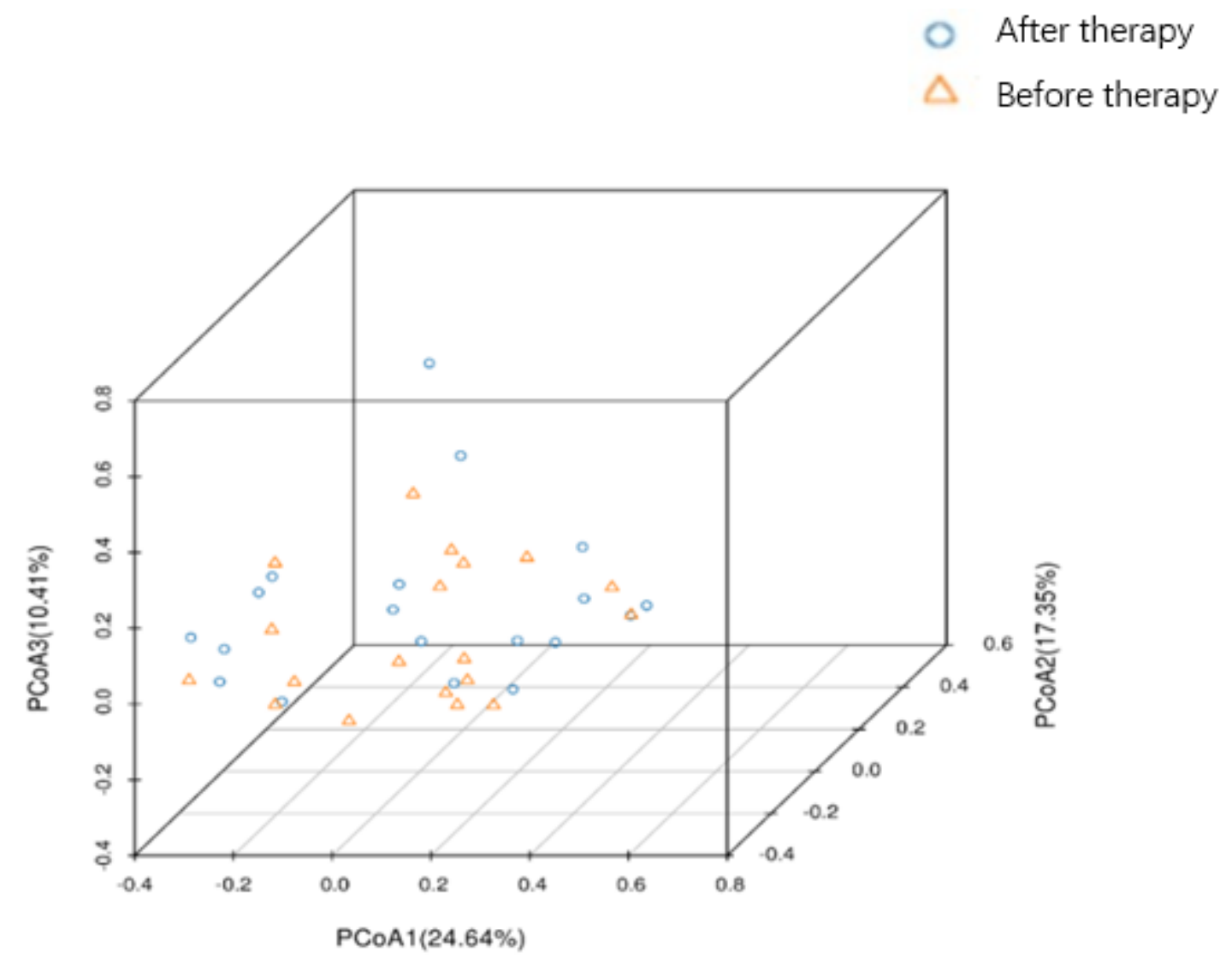

\section{Figure 4}

Principal coordinate analysis (PCOA) of genera using Bray-Curtis dissimilarity analysis. Orange triangles, before treatment (group B1); blue circles, after treatment (group A1). The distances between the two groups indicate that no differences were induced by ACTH therapy.

\section{Supplementary Files}

This is a list of supplementary files associated with this preprint. Click to download.

- cleandata.rar

- table1.pdf 